\title{
Alterations in oleanolic acid and sterol content in marigold (Calendula officinalis) hairy root cultures in response to stimulation by selected phytohormones
}

\author{
Abdulwadood S. M. Alsoufi ${ }^{1}$ - Klaudia Staśkiewicz² $\cdot$ Michał Markowski $^{2}$
}

Received: 20 March 2020 / Revised: 29 July 2020 / Accepted: 18 January 2021 / Published online: 13 February 2021

(c) The Author(s) 2021

\begin{abstract}
Hairy root cultures are an efficient tool for the biotechnological production of plant metabolites and a convenient experimental model for analyzing the effect of various compounds on plant metabolism. In contrast to many other types of in vitro plant cultures, hairy roots do not require an external supply of phytohormones to the medium. Consequently, plant growth regulators such as auxins and cytokinins are rarely used as elicitors in hairy root in vitro cultures; however, they can strongly influence plant defense responses. The aim of this study was to investigate the influence of two auxins: natural indole3-acetic acid (IAA) and synthetic 1-naphthaleneacetic acid (NAA), as well as two cytokinins: natural kinetin and synthetic 6-benzylaminopurine (BAP) at a concentration of $0.75 \mathrm{mg} / \mathrm{L}$ on the metabolism of sterols and triterpenoids in Calendula officinalis hairy roots. Auxins prevented the accumulation of triterpenoid saponins (oleanolic acid glycosides), while cytokinin BAP increased their accumulation by $17 \%$ and their release into the culture medium by a factor of 10 . Other cytokinins and kinetins increased the sterol levels by $17 \%$, the level of stigmasterol by $15 \%$, and the level of isofucosterol by 7 times.
\end{abstract}

Keywords In vitro cultures $\cdot$ Hairy roots $\cdot$ Marigold (Calendula officinalis) $\cdot$ Phytohormones $\cdot$ Triterpenoids

\section{Introduction}

Hairy root cultures (HRCs), induced by infection with Agrobacterium rhizogenes, are plant tissue cultures that are popular in biotechnology due to their numerous advantages. HRCs are easy to maintain on solid or liquid MS (Murashige and Skoog 1962) or other suitable media. Moreover, in contrast to many other types of in vitro plant cultures, they do not require an external supply of phytohormones (plant growth regulators). HRCs have been shown to accumulate valuable phytochemicals at levels comparable to those of intact plants and in higher yields than suspension cell cultures. Their biosynthetic capacities are also usually stable (Ono et al. 2011; Georgiev et al. 2007).

Communicated by M. Lambardi.

Michał Markowski

m.markowski@biol.uw.edu.pl

1 Department of Biology, College of Science, University of Tikrit, Tikrit, Iraq

2 Department of Plant Biochemistry, Faculty of Biology, University of Warsaw, Warsaw, Poland
Plant metabolism can be induced to produce defense metabolites under the influence of different biotic and abiotic elicitors. Indeed, the stimulation of secondary, or specialized, plant metabolism (regarded as a defence mechanism in wild plants) triggered by elicitors is a well-known phenomenon in HRCs (Georgiev et al. 2007; Chandra and Chandra 2011). Biotic elicitors include external substances, those of pathogenic origin (exogenous factors), and compounds synthesized by plants (endogenous factors), e.g., effectors synthesized in response to different stressors, such as jasmonic acid, methyl jasmonate, and salicylic acid. These effectors are plant growth regulators (phytohormones) that are very often applied in HRCs as elicitors. In contrast, plant growth regulators, such as auxins and cytokinins, are rarely used as elicitors in plant tissue cultures, although they can strongly influence plant defense responses (Pieterse et al. 2012).

The aim of this study was to investigate the influence of two auxins: natural indole-3-acetic acid (IAA) and synthetic 1-naphthaleneacetic acid (NAA), as well as two cytokinins: natural kinetin and synthetic 6-benzylaminopurine (BAP) at a concentration of $0.75 \mathrm{mg} / \mathrm{L}$ on the metabolism of sterols and triterpenoids in Calendula officinalis hairy roots. $C$. officinalis wild-type plant and its derived hairy roots produce 
triterpenoid oleanolic acid (OA) and glycosides (saponins) (Niżyński et al. 2015). The main form of OA in both wildtype plant and tissue cultures are glycosides, making $C$. officinalis HRC a suitable model for studying the influence of various elicitors and culture conditions on saponin productivity. The biosynthetic step of 2,3-oxidosqualene cyclization is often regarded as a branch point between primary and secondary metabolism, i.e. between the biosynthesis of primary steroids and secondary/specialized pentacyclic triterpenoids (Moses et al. 2013). In response to various stress factors and elicitors, this branch point can be switched to redirect the metabolic flux towards either steroids or triterpenoids (Alsoufi et al. 2019a,b). Thus, the results of this study can also elucidate whether auxins and cytokinins can act as "switchers" between primary and specialized metabolism.

HRCs do not require the addition of any plant growth regulators to the medium, which is one of their advantages (Georgiev et al. 2007). However, it does not mean that auxins and cytokinins may not have any effect on the metabolism and growth of the culture. Thus, the results obtained in the present study broaden the knowledge on the influence of auxins and cytokinins on hairy root cultures, particularly on triterpenoid production, what can be potentially useful in biotechnological processes.

\section{Materials and methods}

Hairy root culture CC16 was obtained according to a previously described procedure (Długosz et al. 2013). The roots were cultivated in liquid $1 / 2 \mathrm{MS}$ medium, at $23-25{ }^{\circ} \mathrm{C}$, in the darkness on a rotatory shaker at $120 \mathrm{rpm}$. The culture was maintained by subculture every 3-4 weeks.

Freshly subcultured roots were cultured for 16 days to obtain at least $6 \mathrm{~g}$ fresh weight. After they were weighed and transferred to a fresh medium. Five following days after NAA, IAA, kinetin, and BAP were added to a final concentration of $0.75 \mathrm{mg} / \mathrm{L}$. All phytohormones were dissolved in $1 \mathrm{M} \mathrm{KOH}$, diluted by distilled water, and filter-sterilized. The culture was completed after 7 days of incubation with phytohormones.

The initial incubation (16 days) was performed to obtain the relatively high biomass of hairy root cultures that would be sufficient for experiments. According to the previous report (Długosz et al. 2013) the hairy root CC16 line is in the exponential phase of growth on 16th day of culture. Afterwards, the hairy root cultures were transferred to a fresh medium (without cutting root explants) and allowed to grow for 5 days to adapt them to fresh medium conditions, and to stabilize the growth after the transfer. The 7-day period of incubation with a supplemented hormone was chosen in accordance with the previous experiments concerning the influence of various elicitors on triterpenoid metabolism (Alsoufi et al. 2019a, b).

The accumulated dry weight (ADW) $[\mathrm{g} / \mathrm{L}]$ was calculated from dry weight divided by culture medium volume, the growth index (GI) was calculated from fresh weight at the end of the culture and fresh weight at the day of the last subculture as follows:

$G I=\frac{\text { Fresh weight final }- \text { Fresh weight subculture }}{\text { Fresh weight subculture }}$

The harvested hairy roots were dried at room temperature for at least two weeks, and the culture medium was extracted with $n$-butanol. Dried hairy roots were powdered and extracted using a Soxhlet apparatus for $8 \mathrm{~h}$ with diethyl ether and then $8 \mathrm{~h}$ with methanol. The obtained extracts were evaporated to dryness under reduced pressure on a rotatory evaporator-diethyl ether extract (water bath temp. $40{ }^{\circ} \mathrm{C}$ ) and methanol (water bath temp. $60^{\circ} \mathrm{C}$ ).

The ether extract was separated by preparative TLC on $20 \mathrm{~cm} \times 20 \mathrm{~cm}$ glass plates, manually coated with silica gel $60 \mathrm{H}$ (Merck, Darmstadt, Germany). The solvent system chloroform: methanol 97:3 (v/v) was used to develop the plates. Two fractions were obtained as described by Szakiel et al. (2012): triterpene acids (OA) and free steroids. The free steroid fraction was directly analyzed using GC-MS (Agilent Technologies 7890A) while the triterpene acid fraction was methylated with diazomethane.

Methanol extracts from roots and $n$-butanol extracts from the culture medium were hydrolyzed by $11 \% \mathrm{HCl}$ in $70 \%$ methanol for two hours on a heating mantle under reflux. Subsequently, the hydrolysate was diluted with distilled water, methanol was evaporated, and the hydrolysate was diluted with distilled water again. Afterwards, the hydrolysate was extracted three times by diethyl ether $(40 \mathrm{~mL})$ in a separation funnel. The obtained extract was washed with distilled water three times and evaporated to dryness.

The dried extract was divided by preparative TLC on $20 \mathrm{~cm} \times 20 \mathrm{~cm}$ glass plates, manually coated with silica gel $60 \mathrm{H}$ (Merck, Darmstadt, Germany). The solvent system chloroform: methanol 95:5 (v/v) was used for developing the plates. Purified oleanolic acid was methylated with diazomethane.

The samples were dissolved in suitable portions of a diethyl ether: methanol 3:1 $(v / v)$ mixture. Quantitative measurement of oleanolic acid (in the form of its methyl ester) was performed by gas-liquid chromatography (GLC) at $270{ }^{\circ} \mathrm{C}$ on a Shimadzu GC-2014 instrument equipped with a flame ionization detector. Samples were applied by split injection 1:5 on a ZB-1 $30 \mathrm{~m} \times 0.25 \mathrm{~mm} \times 0.25 \mu \mathrm{m}$ column (Phenomenex, SHIM-POL, Izabelin, Poland). The temperature of the injector and detector was $290{ }^{\circ} \mathrm{C}$. Nitrogen was used as the carrier gas at a flow rate of $1.2 \mathrm{~mL} / \mathrm{min}$. Peak 
identification and quantification of oleanolic acid were carried out by referring to a calibration curve prepared with an authenticated sample of methylated oleanolic acid as standard (Wiktorowska et al. 2010).

An Agilent Technologies 7890A gas chromatograph (GC-MS) (Perlan Technologies, Warszawa, Poland) equipped with a 5975C mass selective detector was used for qualitative and quantitative analyses. Samples dissolved in diethyl ether:methanol $(5: 1, \mathrm{v} / \mathrm{v})$ were applied (in a volume of 1-4 $\mu \mathrm{L}$ ) using 1:10 split injection. The column used was a $30 \mathrm{~m} \times 0.25 \mathrm{~mm}$ i.d., $0.25-\mu \mathrm{m}$, HP-5MS UI (Agilent Technologies, Santa Clara, CA, USA). Helium was used as the carrier gas at a flow rate of $1 \mathrm{~mL} / \mathrm{min}$. The separation was made either under isothermal conditions at $280{ }^{\circ} \mathrm{C}$ or with the following temperature program: initial temperature of $160{ }^{\circ} \mathrm{C}$ held for $2 \mathrm{~min}$, then increased to $280^{\circ} \mathrm{C}$ at $5{ }^{\circ} \mathrm{C} / \mathrm{min}$, and the final temperature of $280^{\circ} \mathrm{C}$ held for a further $44 \mathrm{~min}$. The other employed parameters were as follows: inlet and FID (flame ionization detector) temperature $290{ }^{\circ} \mathrm{C}$; MS transfer line temperature $275^{\circ} \mathrm{C}$; quadrupole temperature $150{ }^{\circ} \mathrm{C}$; ion source temperature $230{ }^{\circ} \mathrm{C}$; EI $70 \mathrm{eV} ; \mathrm{m} / z$ range 33-500; FID gas $\left(\mathrm{H}_{2}\right)$ flow $30 \mathrm{~mL} \cdot \mathrm{min}^{-1}$ (hydrogen generator HydroGen PH300, Peak Scientific, Inchinnan, UK); and airflow $400 \mathrm{~mL} \cdot \mathrm{min}^{-1}$. Individual compounds were identified by comparing their mass spectra with library data from Wiley 9th ED. and NIST 2008 Lib. SW Version 2010, or previously reported data, and by comparison of their retention times and corresponding mass spectra with those of authentic standards, where available. Quantitation of steroids was performed using an external standard method based on calibration curves determined for an authentic standard of sitosterol (Woźniak et al. 2018).

For artwork and illustrations, Microsoft Excel and Microsoft Paint were used. For statistical analysis t-Student's test was utilized using Microsoft Excel and STATISTICA by Statsoft Inc. Significance level was set at $\alpha=0,05$. Every variant was done in triplicate.

\section{Results}

The growth parameters of the cultures treated with auxins and cytokinins, expressed as the ADW and GI of $C$. officinalis hairy roots are presented in Fig. 1. Among the tested compounds, only kinetin influenced the growth of hairy roots, which resulted in a $36 \%$ ( $p$ value $=0.18$, not significant) decrease in the fresh weight compared with the untreated control; however, its influence on the ADW was much less pronounced (decreased by only $12 \%$ ), indicating that kinetin mainly changed the degree of hairy root hydration. The effect of other applied phytohormones on the dry weight of hairy roots was less remarkable. None

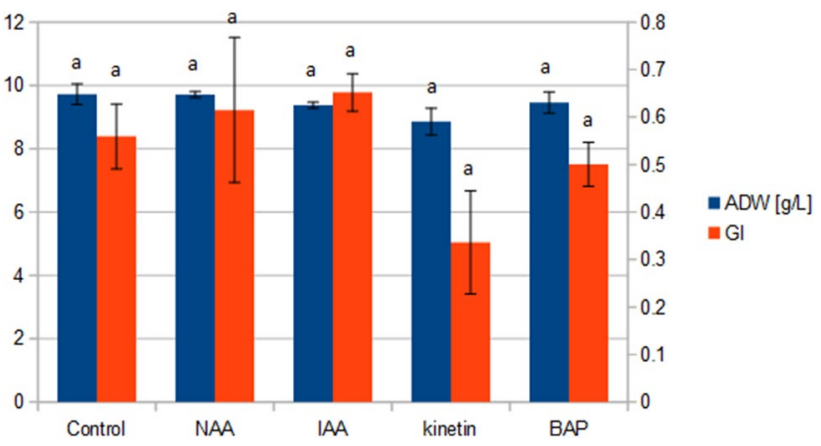

Fig. 1 Accumulated dry weight [g/L] (left) and growth index (right) ( \pm SE, standard error) of hairy roots after 7 days of elicitation with phytohormones compared to the control samples. Different letters indicate significant differences among mean values

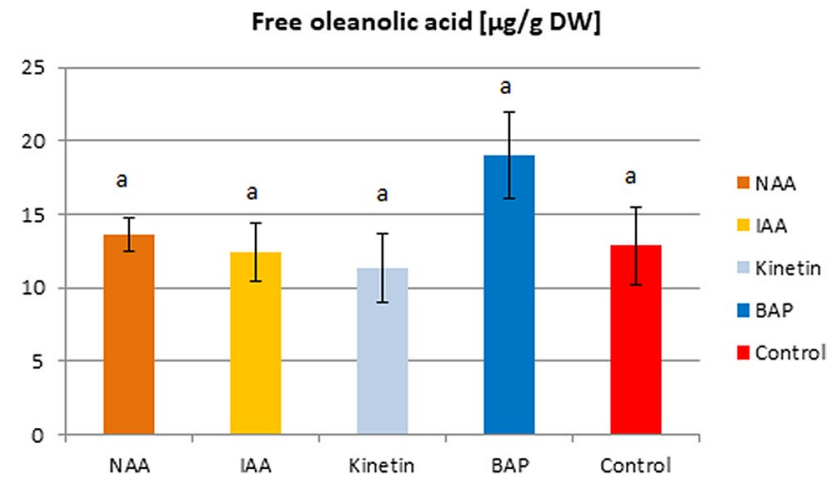

Fig. 2 The content of free oleanolic acid in hairy root tissue $( \pm S E$, standard error) after elicitation phytohormones. Different letters indicate significant differences among mean values

of phytohormones affected root color, morphology, root branching or medium color.

The results concerning the influence of the applied phytohormones on the free and glycosylated oleanolic acid contents, accumulated in hairy root tissues and secreted into the surrounding medium are presented in Figs. 2, 3, 4. The free (non-glycosylated) oleanolic acid accumulated in the hairy root tissues as a saponin precursor increased by $32 \%$ in the presence of BAP (Fig. 2). NAA increased the oleanolic acid content by $6 \%$, whereas IAA and kinetin decreased this level by 4 and $12 \%$, respectively; however, these effects were negligible. In contrast, the influence of all applied phytohormones on the level of glycosylated oleanolic acid (expressed as the content of this compound released after the saponin hydrolysis) accumulated in hairy root tissues was visible and significant (Fig. 3). The accumulation of saponins in hairy roots was markedly decreased by both auxins (NAA by $42 \%$, IAA by $30 \%$ ), as well as kinetin $(20 \%)$. The only factor that increased the saponins content in hairy root tissues was the synthetic cytokinin BAP, although this increase was relatively mild (17\%). Again, the synthetic cytokinin BAP 
Glycosylated oleanolic acid content [mg/g DW]

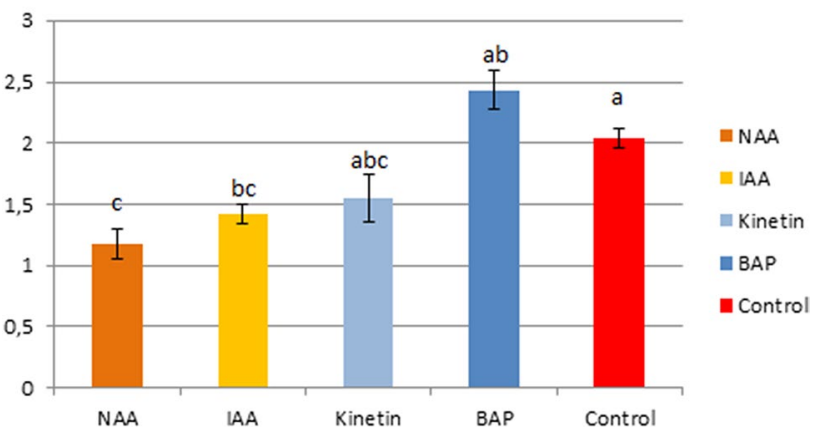

Fig. 3 The content of glycosylated oleanolic acid in hairy root tissue $( \pm$ SE, standard error) after elicitation with phytohormons. Different letters indicate significant differences among mean values

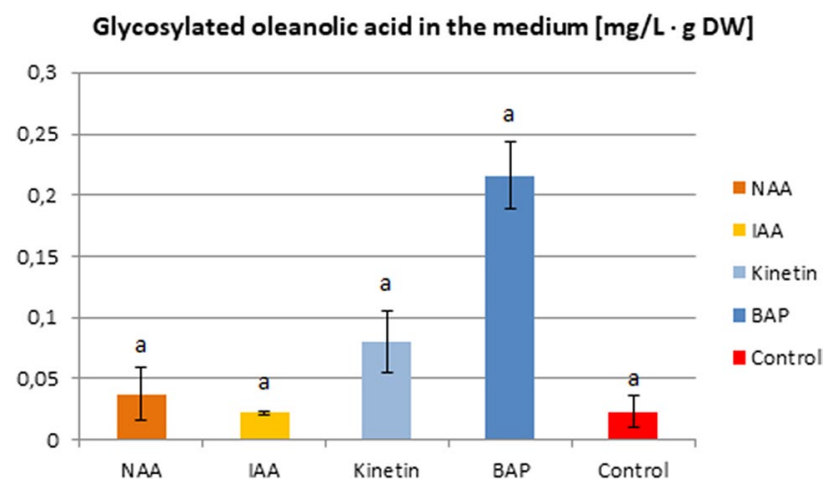

Fig. 4 The concentration of glycosylated oleanolic acid in the medium $( \pm$ SE, standard error) after elicitation with phytohormons. Different letters indicate significant differences among mean values

was the most efficient at increasing the level of saponins secreted into the surrounding medium (Fig. 4). This increase was more than tenfold, which was the strongest influence observed in this study. The natural cytokinin, kinetin, also increased the release of saponins from hairy roots into the surrounding medium, but less significantly than BAP, i.e., fourfold. Neither tested auxin exerted any substantial effect on saponin secretion: the twofold increase exerted by NAA was not statistically significant, and IAA had no observable effect.

The sterol content in hairy root cultures exposed to phytohormones is presented in Table 1. The main sterol profile of C. officinalis hairy roots was composed of cholesterol, campesterol, isofucosterol, sitosterol, and stigmasterol. Other steroids, i.e., an intermediate in the biosynthetic sterol pathway, 24-methylenecycloartanol, the saturated derivative of sitosterol-sitostanol, and the oxidized derivative, ketone, stigmasta-3,5-dien-7-one (tremulone), were also detected. The total content of the main sterols in the control hairy roots was $470 \mu \mathrm{g} / \mathrm{g}$ d.w., and the amount of other detected steroids (stigmastanol, tremulone and 24-methylenecycloartanol) was only $27 \mu \mathrm{g} / \mathrm{g}$ d.w. Kinetin increased the total sterol content by $84 \mu \mathrm{g} / \mathrm{g}$ d.w., mainly due to the elevated amount of the dominating sterol, stigmasterol (by $63 \mu \mathrm{g} / \mathrm{g} \mathrm{d.w.),} \mathrm{and} \mathrm{a} \mathrm{sevenfold} \mathrm{increase} \mathrm{in} \mathrm{the} \mathrm{amount} \mathrm{of}$ isofucosterol. Treatment with BAP and NAA decreased the total sterol content by $78 \mu \mathrm{g} / \mathrm{g}$ d.w. and $59 \mu \mathrm{g} / \mathrm{g} \mathrm{d}$.w., respectively, while IAA had practically no effect.

\section{Discussion}

The growth and general hairy root appearance of $C$. officinalis HRC were carefully observed to detect any possible harmful influence of the applied phytohormones. It has been shown that some elicitors often negatively affect hairy root growth and can change the color and the shape of organs, particularly when applied at higher concentrations (Alsoufi

Table 1 The content of sterols and steroids in Calendula officinalis hairy root tissue after elicitation with phytohormons, mean value \pm SE, standard error

\begin{tabular}{|c|c|c|c|c|c|}
\hline Compound & $\begin{array}{l}\text { Control } \\
\text { [ } \mu \mathrm{g} / \mathrm{g} \text { d.w.] }\end{array}$ & $\begin{array}{l}\text { NAA } \\
\text { [ } \mu \mathrm{g} / \mathrm{g} \text { d.w.] }\end{array}$ & $\begin{array}{l}\text { IAA } \\
\text { [ } \mu \mathrm{g} / \mathrm{g} \text { d.w. }]\end{array}$ & $\begin{array}{l}\text { Kinetin } \\
{[\mu \mathrm{g} / \mathrm{g} \text { d.w. }]}\end{array}$ & $\begin{array}{l}\text { BAP } \\
{[\mu \mathrm{g} / \mathrm{g} \text { d.w. }]}\end{array}$ \\
\hline Cholesterol & $4.56 \pm 1.16 \mathrm{a}$ & $2.01 \pm 0.19 \mathrm{a}$ & $2.29 \pm 0.06 \mathrm{a}$ & $6.02 \pm 1.94 \mathrm{a}$ & $1.49 \pm 0.30 \mathrm{a}$ \\
\hline Campesterol & $35.40 \pm 1.82 \mathrm{a}$ & $26.91 \pm 3.70 \mathrm{a}$ & $31.30 \pm 1.47 \mathrm{a}$ & $32.74 \pm 13.22 \mathrm{a}$ & $23.78 \pm 4.25 \mathrm{a}$ \\
\hline Stigmasterol & $361.20 \pm 18.34 \mathrm{a}$ & $316.00 \pm 44.94 \mathrm{ab}$ & $340.38 \pm 12.03 \mathrm{a}$ & $424.07 \pm 7.89 b$ & $297.86 \pm 40.89 \mathrm{a}$ \\
\hline Sitosterol & $67.59 \pm 1.66 \mathrm{a}$ & $64.52 \pm 7.39 \mathrm{ab}$ & $80.93 \pm 1.20 \mathrm{~b}$ & $72.60 \pm 6.65 \mathrm{ab}$ & $66.76 \pm 10.72 \mathrm{ab}$ \\
\hline Stigmastanol & $12.15 \pm 0.33 a$ & $8.20 \pm 1.29 b$ & $10.57 \pm 0.30 b$ & $11.17 \pm 1.73 \mathrm{ab}$ & $10.72 \pm 1.24 \mathrm{ab}$ \\
\hline Isofucosterol & $1.38 \pm 1.08 \mathrm{a}$ & $1.79 \pm 0.90 \mathrm{a}$ & $4.25 \pm 0.19 \mathrm{a}$ & $19.13 \pm 7.62 \mathrm{a}$ & $1.75 \pm 0,94 \mathrm{a}$ \\
\hline Tremulone & $9.99 \pm 2.50 \mathrm{a}$ & $1.88 \pm 0.43 b$ & $5.45 \pm 1.32 \mathrm{ab}$ & $3.10 \pm 1.37 \mathrm{ab}$ & $2.84 \pm 0.68 \mathrm{ab}$ \\
\hline 24methylenecycloartanol & $5.14 \pm 1.25 \mathrm{a}$ & $8.06 \pm 0.53 \mathrm{ab}$ & $8.86 \pm 0.17 b$ & $7.80 \pm 0.84 \mathrm{ab}$ & $8.10 \pm 0.26 \mathrm{ab}$ \\
\hline Sum of sterols & $470.13 \pm 19.61 \mathrm{ab}$ & $411.24 \pm 55.70 \mathrm{ab}$ & $459.15 \pm 14.23 \mathrm{a}$ & $554.56 \pm 30.62 \mathrm{~b}$ & $391.64 \pm 55.52 \mathrm{ab}$ \\
\hline Sum of other steroids & $497.41 \pm 17.11 \mathrm{a}$ & $429.39 \pm 57.90 \mathrm{a}$ & $484.04 \pm 12.78 \mathrm{a}$ & $576.63 \pm 33.62 \mathrm{a}$ & $413.30 \pm 54.10 \mathrm{a}$ \\
\hline
\end{tabular}

For each line, different letters indicate significant differences among mean values 
et al. 2019b). Therefore, the concentration of phytohormones applied in this study $(0.75 \mathrm{mg} / \mathrm{L})$ was chosen as potentially the safest for the designed experiments. Indeed, the compounds applied as elicitors in this study did not exert a significant effect on root growth, color and morphology, except for kinetin which decreased (by 36\%) the GI of C. officinalis HRC. It was assumed that higher concentrations of the phytohormones, e.g., $1 \mathrm{mg} / \mathrm{L}$, may potentially be too harmful to maintain the undisrupted growth and development of hairy roots.

Among all tested phytohormones, the synthetic cytokinin BAP showed the greatest effect on triterpenoid metabolism, simultaneously increasing both the biosynthesis and the accumulation of free oleanolic acid and its saponins in Calendula hairy roots. Moreover, it also triggered competition between the biosynthetic pathways of sterols and triterpenoids, since an increase in the triterpenoid (oleanolic acid) content was accompanied by a decrease in the sterol content. Surprisingly, the natural cytokinin (kinetin) acted differently and significantly increased the biosynthesis of sterols and the secretion of oleanolic acid saponins, and simultaneously decreased the content of free oleanolic acid and saponins accumulated in hairy root tissue. Thus, it can be concluded that, under particular conditions, natural and synthetic phytohormones may influence specific plant cell metabolism pathways.

In contrast to cytokinins, both natural and synthetic auxins showed similar influences on triterpenoid metabolism, decreasing the content of saponins accumulated in hairy root tissues and secreted into the medium. In addition, they exerted either a slight decrease or practically no effect on sterol biosynthesis. This decreased triterpenoid saponin content under the influence of auxins might have some biotechnological implications for improving hairy root culture productivity. The T-DNA sequence from the Ri (root-inducing) plasmid from Agrobacterium rhizogenes, used for the induction of hairy roots, contains genes for auxin synthesis, iaaM and $i a a H$, but they are not necessary for forming HRC. The genes responsible for the formation of hairy roots inserted in T-DNA are rol family: rolA, rolB, rolC, and rolD (Gieorgiev et al. 2007). The deletion or silencing of the iaaM and iaaH from T-DNA may create more efficient $C$. officinalis hairy root lines in terms of the production of glycosylated oleanolic acid.

Cytokinins can influence plant defence responses such as salicylic acid accumulation, increased virus resistance, and general influences on biotrophic pathogen defense (Choi et al. 2011; Pieterse et al. 2012). The increased synthesis of glycosylated oleanolic acid, as well as the increased stigmasterol content under the influence of kinetin, may have been due to the defence-related effect of these phytohormones. Auxins suppress salicylic acid levels and signaling, so if the effect of cytokinins on triterpenoid accumulation is due to their positive influence on salicylic acid pathways, auxins are expected to exert the reverse effect (Pieterse 2012).

In a previous study, $C$. officinalis HRCs were cultivated in media containing different nitrogen sources. The main observed effects were: a better hairy root growth when nitrate was the predominant nitrogen source, a lower edamine content in the medium, and a possible six-fold increase in the biosynthesis of oleanolic acid glycosides when the ammonium-to-nitrate ratio was changed (Długosz et al. 2018). Apart from the nitrogen source, various biotic and abiotic elicitors can also influence the saponin production in C. officinalis HRCs (Alsoufi et al. 2019a, b). The most efficient elicitor applied thus far was jasmonic acid, whereas the effects of chitosan and various abiotic factors were much less pronounced. This study is the first report concerning the treatment of hairy roots with auxins and cytokinins. Although these compounds were not supplied to HRCs routinely as elicitors, our results show that they can influence the metabolism of hairy roots and the resulting productivity of this type of in vitro plant culture.

\section{Conclusions}

This study represents the first research concerning the effect of auxins and cytokinins on hairy roots, particularly concerning triterpenoid production. The results showed that phytohormones did not harm marigold hairy root cultures at the applied concentration, which indicates that plant phytohormones are safe for hairy roots, even when their hormonal balance is changed by T-DNA.

Saponin content in hairy root cultures and their release into the culture medium were increased by one of the cytokinins-BAP. This might indicate that this phytohormone can be used as an effective elicitor for this system, and it can be hypothesized that the defensive reaction of the plant resulting in an increased production of oleanolic acid glycosides involves cytokinins. The examined auxins showed the opposite effect, and the accumulation and release of saponins decreased. This might lead to a new biotechnological strategy, in which the Ri plasmid T-DNA is changed by cutting out iaaM and iaaH (synthesis of IAA) (Gieorgiev et al. 2007) to possibly increase the production of triterpenoids.

Phytosterols were markedly affected by one of the investigated phytohormones-kinetin. This influence particularly concerned stigmasterol (17\% increase) and isofucosterol (sevenfold increase). The strong competition between pentaand tetracyclic triterpenoids was not observed in this study.

Author contribution statement Conceived and designed the experiments: ASMA, MM. Performed the experiments: ASMA, KS, MM. Analyzed the data: ASMA, KS, MM. Contributed reagents/materials/analysis tools: ASMA, 
MM. Wrote the paper: ASMA, MM. All authors read and approved the final manuscript.

\section{Compliance with ethical standards}

Conflict of interest No conflict of interest.

Open Access This article is licensed under a Creative Commons Attribution 4.0 International License, which permits use, sharing, adaptation, distribution and reproduction in any medium or format, as long as you give appropriate credit to the original author(s) and the source, provide a link to the Creative Commons licence, and indicate if changes were made. The images or other third party material in this article are included in the article's Creative Commons licence, unless indicated otherwise in a credit line to the material. If material is not included in the article's Creative Commons licence and your intended use is not permitted by statutory regulation or exceeds the permitted use, you will need to obtain permission directly from the copyright holder. To view a copy of this licence, visit http://creativecommons.org/licenses/by/4.0/.

\section{References}

Alsoufi ASM, Pączkowski C, Szakiel A, Długosz M (2019a) Effect of jasmonic acid and chitosan on triterpenoid production in Calendula officinalis hairy root cultures. Phytochem Lett 31:5-11. https ://doi.org/10.1016/j.phytol.2019.02.030

Alsoufi ASM, Pączkowski C, Długosz M, Szakiel A (2019b) Influence of selected abiotic factors on triterpenoid biosynthesis and saponin secretion in marigold (Calendula officinalis $\mathrm{L}$.) in vitro hairy root cultures. Molecules 24:2907. https://doi.org/10.3390/ molecules24162907

Chandra S, Chandra R (2011) Engineering secondary metabolite production in hairy roots. Phytochem Rev 10:371. https://doi. org/10.1007/s11101-011-9210-8

Choi J, Choi D, Lee S, Ryu CM, Hwang I (2011) Cytokinins and plant immunity: old foes or new friends? Trends Plant Sci 16:388-394. https://doi.org/10.1016/j.tplants.2011.03.003

Długosz M, Wiktorowska E, Wiśniewska A, Pączkowski C (2013) Production of oleanolic acid glycosides by hairy root established cultures of Calendula officinalis L. Acta Biochim Pol. https://doi. org/10.18388/abp.2013_2008

Długosz M, Markowski M, Pączkowski C (2018) Source of nitrogen as a factor limiting saponin production by hairy root and suspension cultures of Calendula officinalis L. Acta Physiol Plant 40:35. https ://doi.org/10.1007/s11738-018-2610-2
Georgiev MI, Pavlov AI, Bley T (2007) Hairy root type plant in vitro systems as sources of bioactive substances. Appl Microbiol Biotechnol 74:1175. https://doi.org/10.1007/s00253-007-0856-5

Moses T, Pollier J, Thevelein JM, Goossens A (2013) Bioengineering of plant (tri) terpenoids: from metabolic engineering of plants to synthetic biology in vivo and in vitro. New Phytol 200:27-43. https://doi.org/10.1111/nph.12325

Murashige T, Skoog F (1962) A revised medium for rapid growth and bioassays with tobacco tissue cultures. Physiol Plantarum $15: 473-497$

Niżyński B, Alsoufi ASM, Pączkowski C, Długosz M, Szakiel A (2015) The content of free and esterified triterpenoids of the native marigold (Calendula officinalis) plant and its modifications in in vitro cultures. Phytochem Lett 11:410-417. https://doi. org/10.1016/j.phytol.2014.12.017

Ono NN, Tian L (2011) The multiplicity of hairy root cultures: prolific possibilities. Plant Sci 180:439-446. https://doi.org/10.1016/j. plantsci.2010.11.012

Pieterse CM, Van der Does D, Zamioudis C, Leon-Reyes A, Van Wees SC (2012) Hormonal modulation of plant immunity. Annu Rev Cell Dev Biol 28:489-521. https://doi.org/10.1146/annurev-cellb io-092910-154055

Szakiel A, Ruszkowski D, Janiszowska W (2005) Saponins in Calendula officinalis L.-structure, biosynthesis, transport and biological activity. Phytochem Rev 4:151-158. https://doi.org/10.1007/ s11101-005-4053-9

Szakiel A, Pączkowski C, Huttunen S (2012) Triterpenoid content of berries and leaves of bilberry Vaccinium myrtillus from Finland and Poland. J Agric Food Chem 60:11839-11849. https://doi. org/10.1021/jf3046895

Wiktorowska E, Długosz M, Janiszowska W (2010) Significant enhancement of oleanolic acid accumulation by biotic elicitors in cell suspension cultures of Calendula officinalis L. Enzyme Microb Technol 46:14-20. https://doi.org/10.1016/j.enzmi ctec.2009.09.002

Woźniak Ł, Szakiel A, Pączkowski C, Marszałek K, Skąpska S, Kowalska H, Jędrzejczak R (2018) Extraction of triterpenic acids and phytosterols from apple pomace with supercritical carbon dioxide: impact of process parameters, modelling of kinetics, and scalingup study. Molecules 23:2790. https://doi.org/10.3390/molecules2 3112790

Publisher's Note Springer Nature remains neutral with regard to jurisdictional claims in published maps and institutional affiliations. 